Tohoku J. Exp. Med., 2008, 216, 47-52

\title{
Early Diagnosis of Cancer by Detecting the Chemiluminescence of Hematoporphyrins in Peripheral Blood Lymphocytes
}

\author{
Masahiro Kohno, ${ }^{1}$ Motohiro Takeda, ${ }^{2}$ Yoshimi Niwano, ${ }^{1}$ Rumiko Saito, ${ }^{1}$ \\ Natsumi Emoto, ${ }^{1}$ Mika Tada, ${ }^{1}$ Takemichi Kanazawa, ${ }^{3}$ Noriaki Ohuchi ${ }^{2}$ and \\ RIE YAMADA ${ }^{4}$ \\ ${ }^{1}$ New Industry Creation Hatchery Center, Tohoku University, Sendai, Japan \\ ${ }^{2}$ Division of Surgical Oncology, Graduate School of Medicine, Tohoku University, Sendai, Japan \\ ${ }^{3}$ Medical Corporation Murakami Hospital, Aomori, Japan \\ ${ }^{4}$ Tohoku Electronic Industrial Co., Ltd., Sendai, Japan
}

\begin{abstract}
Early detection and optimal treatment are the most effective means to improve cancer mortality. Mass screening for cancer has yielded a marked reduction of cancer mortality in the United States. Simple and effective methods are expected for screening of malignancy. Hematoporphyrin derivatives (HPDs) are known to accumulate in cancer cells; thus, HPD has been used for local diagnosis and photodynamic therapy of cancer. The lymphocytes of cancer patients also demonstrate the active uptake of HPD and this phenomenon has been applied for the diagnosis of cancer. In the present study, we have developed a novel method for measurement of the chemiluminescence of HPD in peripheral blood lymphocytes. HPD is composed of hematoporphyrin and its oligomers. Seven cancer patients and seven controls were recruited for this study. The primary cancers included two prostate cancers (one without metastasis and the other with lung metastasis), a renal cancer, a lung adenocarcinoma with systemic metastasis, two gallbladder cancers with lung metastasis, and a colon cancer with liver metastasis. HPD in lymphocytes was measured using a highly sensitive chemiluminescence analyzer with laser light irradiation to detect photoemission by ${ }^{1} \mathrm{O}_{2}$ from HPD. The intensity of chemiluminescence exhibited a linear correlation with the concentrations of HPD. In addition, the level of HPD in lymphocytes was significantly higher in cancer patients than that in healthy volunteers $(p<0.05)$. These results suggest that detection of the chemiluminescence of HPD in lymphocytes could be a sensitive and simple method for cancer diagnosis and screening. - Cancer; hematoporphyrin derivative; chemiluminescence analyzer; singlet oxygen; lymphocyte.
\end{abstract}

Tohoku J. Exp. Med., 2008, 216 (1), 47-52.

(C) 2008 Tohoku University Medical Press

Photodynamic therapy involves the selective uptake and retention of photosensitizing agents for the diagnosis and treatment of malignancies (Lipson et al. 1961; Cortese et al. 1979; Hayata et al. 1982). The accumulation of photosensitizing agents and their activation by a specific wavelength of light (Dougherty et al. 1975; Gomer et al. 1979; Dougherty 1987) lead to the formation

Received February 29, 2008; revision accepted for publication July 15, 2008.

Correspondence: Masahiro Kohno, Ph.D. New Industry Creation Hatchery Center, Tohoku University, Aoba 6-6-10, Aramaki, Aoba-ku, Sendai, 980-8579, Japan.

e-mail:mkohno@niche.tohoku.ac.jp 
of an excited triplet state and chain reactions of reactive oxygen species (ROS); singlet oxygen $\left({ }^{1} \mathrm{O}_{2}\right)$, superoxide $\left(\mathrm{O}_{2}^{-}\right)$, hydrogen peroxide $\left(\mathrm{H}_{2} \mathrm{O}_{2}\right)$ and hydroxyl radical (HO) can selectively target tumor tissues (Peterson et al. 1981; Pótó et al. 1989; Ando et al. 1997). Therefore, measuring the uptake of hematoporphyrin derivative (HPD) in cancerous tissue has been applied to the diagnosis of cancer. Up to now, among the potential activities of HPD for various types of cells, the interaction between peripheral blood lymphocytes and HPD has been also intensively investigated. Its clinical application for screening and followup after cancer therapy has been attempted by measuring the electron spin resonance (ESR) and fluorescence spectrum. Previous studies have attempted to measure the uptake of HPD by fluorescence for cancer screening and follow-up after cancer therapy. However, the nonspecific fluorescence of peripheral blood lymphocytes and insufficient sensitivity of photo-detectors prevented the detection of small amounts of HPD in lymphocytes (Itabashi et al. 1984; Docchio et al. 1984; He et al. 1989; Tanielian et al. 2001).

Recent refinements in photo-detectors and measurement methods enabled the detection of very small amounts of HPD $(<10 \mu \mathrm{g} / \mathrm{ml})$. The fluorescence detection system was composed of a highly sensitive photon counting system and a laser. The amount of HPD is measured by the light intensity as photon counts from ${ }^{1} \mathrm{O}_{2}$ generated from HPD by laser irradiation.

Cancer is a major cause of mortality. Early detection and proper treatment are the most effective means to reduce cancer mortality. Mass screening is essential to identify cancer patients in early stages. Although mass screenings have markedly reduced cancer mortality in the United States, they are expensive and require extensive effort and time. Therefore simple and cost-effective methods are required for cancer screening, as well as for diagnosis and treatment.

In this report, we examined the uptake of HPD by peripheral blood lymphocytes of cancer patients and healthy volunteers by using a novel method for measurement of the chemiluminescence of HPD.

\section{Materials And Methods}

Chemicals

Hematoporphyrin (HP) was purchased from Sigma Chemical Company (St. Louis, MO, USA) and HPD was purchased from Seikagaku Kogyo Co., Ltd. (Tokyo, Japan). The elements of HPD were HP and its oligomers. The average molecular mass of HPD was about 3,000 Da. HPD was diluted in saline to be a concentration of $150 \mathrm{mg} / 30 \mathrm{ml}$. The final concentration of HPD was about $1.66 \mathrm{mM}$. HP was prepared in saline at a concentration of $5 \mathrm{mM}$. Ficoll-Paque PLUS was purchased from GE Healthcare Biosciences AB (Uppsala, Sweden).

\section{Participants}

Seven healthy volunteers and seven cancer patients were recruited at Tohoku University Hospital and Medical Corporation Murakami Hospital (Aomori, Japan). All of the participants were carefully questioned to determine whether they had any malignancies or inflammatory diseases. The primary cancer patient group included two prostate cancers (one was negative for metastasis and the other was positive for lung metastasis), a renal cancer with anemia, a lung adenocarcinoma with systemic metastasis, two gallbladder cancers with lung metastasis and a colon cancer with liver metastasis.

\section{Isolation of lymphocytes}

Lymphocytes were isolated from the venous blood by density gradient centrifugation on Ficoll-Paque PLUS. Isolated lymphocytes were diluted to $5 \times 10^{6}$ cells $/ \mathrm{ml}$ with saline. For HPD-staining, $1 \times 10^{6}$ lymphocytes were treated with $100 \mu 1$ of $1.66 \mathrm{mM}$ HPD solution for $10 \mathrm{~min}$ at $37^{\circ} \mathrm{C}$. Then the lymphocytes were washed twice in phosphate-buffered saline (PBS) and resuspended in $100 \mu 1$ of saline. Fifty $\mu 1$ of the suspension of lymphocytes stained with HPD was used for the chemiluminescence $(\mathrm{CL})$ measurements.

\section{Measurement setup and analytical procedures}

The detection of CL was performed using a chemiluminescence analyzer (CLA) (MLA-GOLDS; Tohoku Electronic Industrial Co., Ltd., Sendai, Japan) which consisted of a photomultiplier tube (R550P, Hamamatsu Photonics, Shizuoka, Japan), a semiconductor laser (VIOLET LASER DIODE: NDHV310APC; Nichia Corporation, Tokushima, Japan) with a wavelength at $408 \mathrm{~nm}$ and a long pass filter of $600 \mathrm{~nm}$. To minimize cell damage, the output energy of the laser was controlled at $10 \mathrm{~mW}$. 
The intensity of the CL of lymphocytes was measured for lymphocytes with HPD and lymphocytes without HPD under laser irradiation at $408 \mathrm{~nm}$. The intensity of the CL of HPD in the lymphocytes was assessed by subtracting the CL of lymphocytes without HPD from the CL of those with HPD. Each assay was performed in duplicate.

\section{Statistical analysis}

The significance of the difference between cancer patients and healthy volunteers was assessed by Student's $t$-test and values of $P<0.05$ were considered to be significant.

This study was approved by the Ethics Committee of Tohoku University.

\section{Results}

The representative emission spectra observed in lymphocytes of a colon cancer patient with and without HPD staining are shown in Fig. 1. The emission spectrum of HPD in lymphocyte of cancer patients exhibited two peaks at 560 580 nm and $620 \sim 640 \mathrm{~nm}$. One of these peaks at 620 640 $\mathrm{nm}$ was not observed in the lymphocytes of cancer patients without HPD. This is consistent with the previous finding that emission in the range of $620 \sim 640 \mathrm{~nm}$ is caused by ${ }^{1} \mathrm{O}_{2}$.

The CL intensity of HP was measured at several concentrations to make a calibration curve and to determine the detection limit of HPD in lymphocytes. The CL intensities in the range over $600 \mathrm{~nm}$ showed a strong positive correlation with the HP concentrations (Fig. 2). The correlation coefficient was 0.9951 . Since the specific molecular structure, mass and composition of the HPD is unclear, a calibration curve of CL of single HP was used to determine the concentration of the HPD. The detection limit of the CLA for HPD was determined to be lower than 1 pmol (Fig. 2B). The sensitivity of the CLA was 1000-fold higher than that of the commercially available fluorescence spectrometry.

Fig. 3 shows the intensity of CL from the lymphocytes of cancer patients and of healthy volunteers. A nonspecific CL of 5,000-7,000 cps was observed in the lymphocytes of all participants without HPD. Therefore, the specific CL of ${ }^{1} \mathrm{O}_{2}$ was calculated by subtraction of the CL without HPD from the CL with HPD (Fig. 4). The average HPD concentration in the lymphocytes of cancer patients was estimated to be $100 \mathrm{pmol}$ in $1.25 \times 10^{5}$ cells using the calibration curve (Fig. 2). The CL intensity from the lymphocytes of cancer patients was significantly higher than that of the lymphocytes from healthy volunteers (Fig. 3, $p<$ $0.05)$.

\section{Discussion}

A significant uptake of HPD was observed in

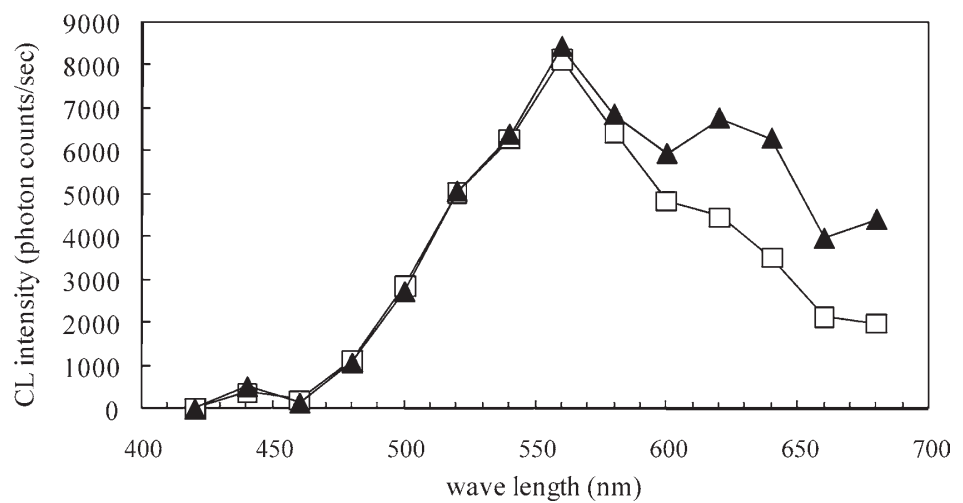

Fig. 1. Fluorescence spectra of the lymphocytes of a colon cancer.

$\Delta$ : Fluorescence with HPD staining $\square$ : Fluorescence without HPD staining

The line with $\square$ indicates the fluorescence spectrum of the lymphocytes from a colon cancer without HPD. The line with $\boldsymbol{\Delta}$ indicates the fluorescence spectrum of the lymphocytes from a colon cancer with HPD. 
A

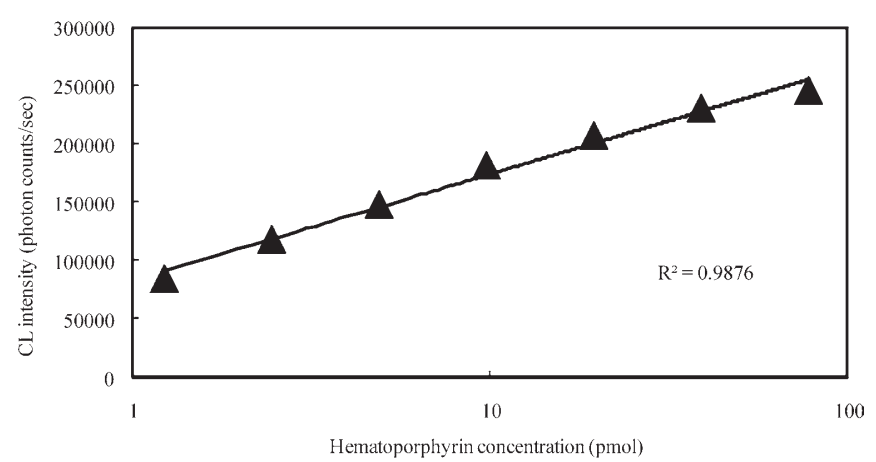

B

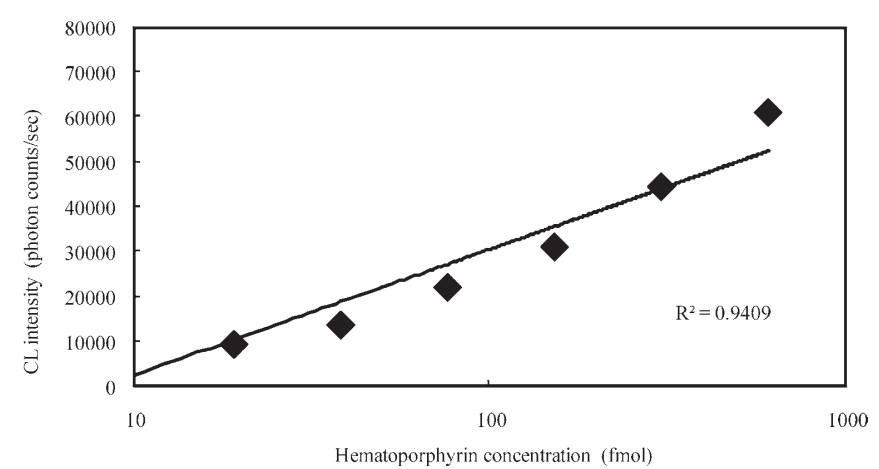

Fig. 2. Calibration curve of the standard HP concentration and chemiluminescence intensity.

A. Correlation between the fluorescence intensity and HP in the high concentration range (1 to 100 pmol).

B. Correlation between the fluorescence intensity and HP in the low concentration range (10 to 1,000 fmol).

the peripheral blood lymphocytes of all cancer patients by subtraction of the emission intensity from that before the treatment with HPD. In contrast, there was no significant difference between the emission intensity before and after the treatment with HPD in normal volunteers. These results suggest that the selective uptake of HPD by lymphocytes can be applied for the diagnosis and screening of cancer.

Fluorescence measurements have been used for the diagnosis of various diseases (Kinoshita et al. 1988). In particular, the measurement of ultra weak photon emission has been used to study the biological activity of leukocytes and to find biomarkers in whole blood that are related to aging (Inaba et al. 1979; Dodeigne et al. 2000). The fluorescence measurement of peripheral blood lymphocytes stained with HPD has not been used for clinical applications because of the nonspe- cific fluorescence of lymphocytes and poor sensitivity of existing florescence spectrometers (Itabashi et al. 1984; Docchio et al. 1984; He et al. 1989; Tanielian et al. 2001). It was possible to discriminate the luminescence of ${ }^{1} \mathrm{O}_{2}$ from the nonspecific $\mathrm{CL}$ of lymphocytes by measuring the CL within the range of $620 \sim 640 \mathrm{~nm}$ with a highly sensitive CLA system.

Although the precise mechanism controlling the selective uptake of HPD in lymphocytes of cancer patients is still unknown, HPD has been reported to accumulate in the hydrophobic region of B cells and NK cells but not T cells (Kessel et al. 1983; Berki et al. 1998; Savitskii, et al. 2004). Therefore, it has been hypothesized that populations of cells with a high affinity to HPD, such as B cells and NK cells, may show a relative increase among the lymphocytes in cancer patients. The lymphocyte profile should therefore 


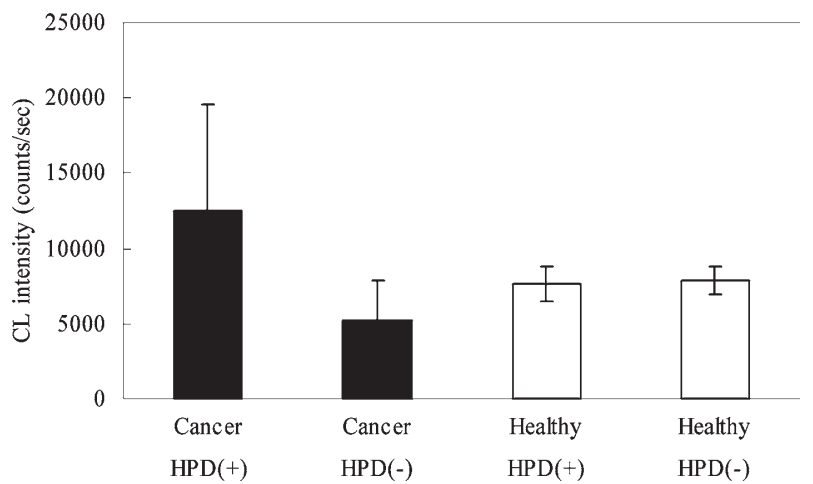

Fig. 3. Intensity of chemiluminescence from lymphocytes of cancer patients and of healthy volunteers.

+: Fluorescence with HPD staining

-: Fluorescence without HPD staining

The black columns indicate the CL intensity of the lymphocytes from cancer patients with and without positive staining for HPD $(n=7)$. The white columns indicate the chemiluminescence of lymphocytes from healthy volunteers with and without positive staining for $\operatorname{HPD}(n=7)$.

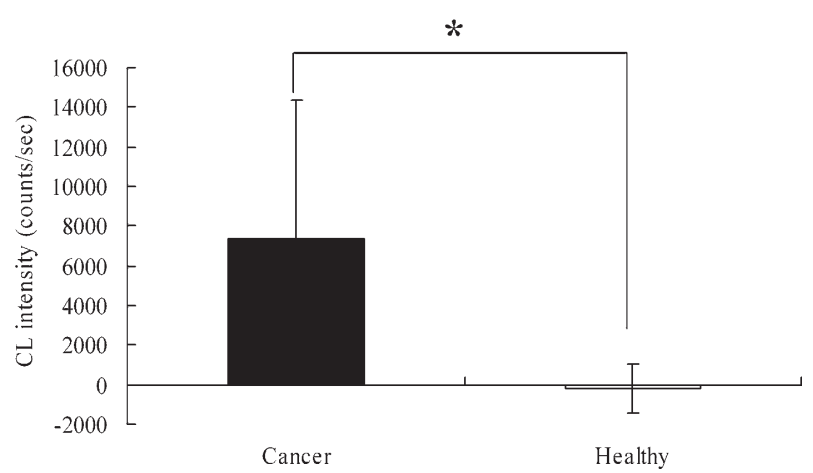

Fig. 4. Chemiluminescence from lymphocytes of cancer patients and of healthy volunteers.

The CL intensity of HPD was calculated by subtraction of the CL without HPD from the CL with HPD for each participant.

*: Statistical significance between cancer patients and healthy volunteers $(p<0.05)$.

be examined as a next step.

In this study, a high uptake of HPD was observed in some types of malignancies. The results suggest that this novel method could be used for mass screening as a simple and costeffective method to detect malignancies.

This method could also serve as an adjunct to non-invasive diagnosis by positron emission tomography, magnet resonance imaging system or $\mathrm{X}$-ray CT.

\section{References}

Ando, T., Yoshikawa, T., Tanigawa, T., Kohno, M., Yoshida, N. \& Kondo, M. (1997) Quantification of singlet oxygen from hematoporphyrin derivative by electron spin resonance. Life Sci., 61, 1953-1959.

Berki, T. \& Németh, P. (1998) Novel method for in vitro depletion of T cells by monoclonal antibody- targeted photosensitization. J. Immunol. Methods, 211, 139-146.

Cortese, D.A., Kinsey, J.H., Woolner, L.B., Payne, W.S., Sanderson, D.R. \& Fontana, R.S. (1979) Clinical application of a new endoscopic technique for detection of in situ bronchial carcinoma. Mayo Clin. Proc., 54, 635-641.

Docchio, F., Ramponi, R., Sacchi, C.A., Bottiroli, G. \& Freitas, I. (1984) Time-resolved fluorescence spectroscopy of hematoporphyrin-derivative in human lymphocytes. Chem. Biol. Interact., 50, 135-141.

Dodeigne, C., Thunus, L. \& Lejeune, R. (2000) Chemiluminescence as diagnostic tool. A review. Talanta, 51, 415-439.

Dougherty, T.J. (1987) Photosensitizers: Therapy and detection of malignant tumors. Photochem. Photobiol., 45, 879-889.

Dougherty, T.J., Grindey, G.B., File, R., Weishaupt, K.R. \& Boyle, D.G. (1975) Photoradiation therapy. II, Cure of animal tumors with hematoporphyrin and light. J. Natl. Cancer Inst., 55, 115-121.

Gomer, C.J. \& Dougherty, T.J. (1979) Determination of $\left[{ }^{3} \mathrm{H}\right]-$ and $\left[{ }^{14} \mathrm{C}\right]$ hematoporphyrin derivative distribution in malignant and normal tissue. Cancer Res., 39, 146-151.

Hayata, Y., Kato, H., Ono, J., Matsushima, Y., Hayashi, N., Saito, T. \& Kawate, N. (1982) Fluorescence fiberoptic bronchoscopy in the diagnosis of early stage lung cancer. Recent Results Cancer Res., 82, 121-130.

He, D., Soter, N.A. \& Lim, H.W. (1989) The late phase of hematoporphyrin derivative- induced phototoxicity in mice: release of histamine and histologic changes. Photochem. Photobiol., 50, 91-95.

Inaba, H., Shimizu, Y., Tsuji, Y. \& Yamagishi, A. (1979) Photon counting spectral analyzing system of extra-weak, chemiand bioluminescence for biological applications. Photochem. Photobiol., 30, 169-175.

Itabashi, M., Aoyama, M., Kohno, M. \& Tanaka, N. (1984) ESR (electron spin resonance) analysis of cancer patient lymphocytes stained with hematoporphyrin D. Gan No Rinsho, 30, 1293-1298.

Kessel, D. \& Chou, T.H. (1983) Tumor-localizing components of the porphyrin preparation hematoporphyrin derivative. Cancer Res., 43, 1994-1999.

Kinoshita, S., Seki, T., Liu, T.F. \& Kucida, T. (1988) Fluorescence of hematoporphyrin in living cell and in solution. $J$. Photochem. Photobiol. B: Biol., 2, 195-208.

Lipson, R.L., Baldes, E.J. \& Olson, A.M. (1961) The use of a derivative of hematoporphyrin in tumor detection. J. Natl. Cancer Inst., 26, 1-11.

Peterson, D.A., Mckelvey, S. \& Edmondson, P.R. (1981) A hypothesis for the molecular mechanism of tumor killing by porphyrins and light. Med. Hyphotheses, 7, 201-205.

Pótó, L. \& Berki, T. (1989) Investigation on the free radical producing effect of hematoporphyrin (a spin trapping study). Acta Physiol. Hung., 74, 285-290.

Savitskii, V.P., Zorin, V.P., Potapnev, M.P. \& Potapenko, A.Y. (2004) Comparative analysis of accumulation of chlorine 
e6 and hematoporphyrin derivatives in subpopulations of peripheral blood lymphocytes. Bull. Exp. Biol. Med., 138, 158-162.

Tanielian, C., Schweitzer, C., Mechin, R. \& Wolff, C. (2001)
Quantum yield of singlet oxygen production by monomeric and aggregated forms of hematoporphyrin derivative. Free Radic. Biol. Med., 30, 208-212. 